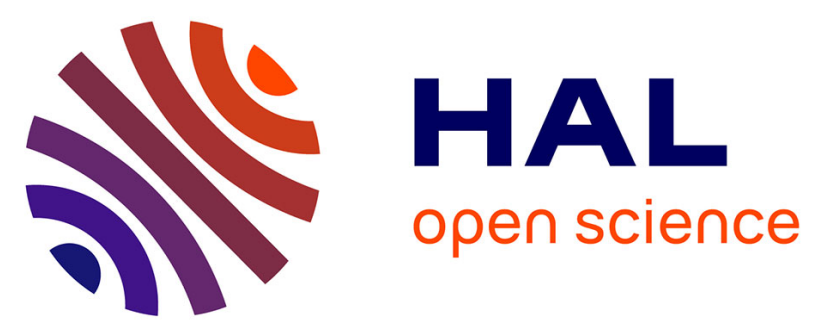

\title{
Generic model of the molecular orientational distribution probed by polarization-resolved second-harmonic generation
}

Julien Duboisset, Dora Ait-Belkacem, Muriel Roche, Hervé Rigneault, Sophie Brasselet

\section{To cite this version:}

Julien Duboisset, Dora Aït-Belkacem, Muriel Roche, Hervé Rigneault, Sophie Brasselet. Generic model of the molecular orientational distribution probed by polarization-resolved second-harmonic generation. Physical Review A: Atomic, molecular, and optical physics [1990-2015], 2012, 85, pp.043829. 10.1103/PhysRevA.85.043829 . hal-00690339

\section{HAL Id: hal-00690339 \\ https://hal.science/hal-00690339}

Submitted on 23 Apr 2012

HAL is a multi-disciplinary open access archive for the deposit and dissemination of scientific research documents, whether they are published or not. The documents may come from teaching and research institutions in France or abroad, or from public or private research centers.
L'archive ouverte pluridisciplinaire HAL, est destinée au dépôt et à la diffusion de documents scientifiques de niveau recherche, publiés ou non, émanant des établissements d'enseignement et de recherche français ou étrangers, des laboratoires publics ou privés. 


\title{
Generic model of the molecular orientational distribution probed by polarization-resolved Second Harmonic Generation
}

\author{
Julien Duboisset*, Dora Aït-Belkacem, Muriel Roche, Hervé Rigneault, Sophie Brasselet* \\ Institut Fresnel, CNRS, Aix-Marseille Université, Ecole Centrale Marseille, \\ Domaine Universitaire St Jérôme, 13013 Marseille, France
}

(Dated: March 16, 2012)

\begin{abstract}
In this work we investigate a generic method able to extract information on molecular organization in biological samples from polarized Second Harmonic Generation (SHG) microscopy, without the need to infer an a priori model for the molecular orientational distribution. The mean orientation of this distribution, as well as its first and third orders of symmetry, are estimated by monitoring SHG intensity signals under a varying incident polarization. We introduce in particular a reduction of the problem to a $2 \mathrm{D}$ approach appropriate to the microscopy geometry. This method permits to retrieve determining information which is not available in the traditional model-oriented methods, as illustrated in molecular order imaging in collagen fibrils. The precision of the parameters estimation is evaluated by a Monte Carlo analysis, based on the Poisson noise statistics of the measured signal.
\end{abstract}

PACS numbers: 42.65.An;78.20.-e;87.14.em

\footnotetext{
* julien.duboisset@fresnel.fr, sophie.brasselet@fresnel.fr
} 


\section{INTRODUCTION}

Second Harmonic Generation (SHG) microscopy, since its first development [1-3] and its introduction in bio-imaging [4-6], is now widely used to image ordered bio-molecular assemblies in complex samples. Coherent SHG occurring naturally in non-centrosymmetric structures such as collagen [3], skeletal muscles [4] and microtubules [6], is today exploited as a functional contrast $[7,8]$, possibly in conjunction with other nonlinear optical signatures [9], with the ultimate goal of developing diagnostics of pathological effects related to tissues and cell architecture. In addition to their unique imaging capabilities, nonlinear contrasts are dependent on the incident light polarization state, providing an interesting way to probe molecular orientation and order. Structural information, from membrane architecture and proteins aggregates to biopolymers and tissue assemblies, is a key parameter in a large variety of biological phenomena. Polarization sensitive imaging has been exploited a long time ago in fluorescence [10, 11] and SHG [12] in ordered molecular samples. SHG polarization resolved imaging has allowed retrieving molecular orientation and order information in molecular materials $[13,14]$ and crystals down to the nanometric size [15], as well as collagen and muscle structural quantitative information in tissues [16-21].

Deducing a structural information (molecular orientational order) from SHG polarization resolved data in microscopy has been so far based on the use of a priori known models in the orientation distribution of the probed system. Polarized SHG applied to collagen type I has shown that its hexagonal crystalline structure can be reduced to an equivalent orientational distribution with a cone surface shape [19-21], similar to what would be obtained from a collection of active molecules assembled along the triple helix structure of the collagen protein. Many works have developed analyzes in this direction, using as an unknown parameter the aperture of this cone to quantify molecular order in collagen assemblies. In complex environments however such as tissues or micrometric assemblies of fibrils, much more complex orientational behavior can be expected. It is delicate to infer a model in this case, although tentative analyzes have been performed in this direction introducing an additional disorder parameter in the surface cone aperture [19]. Adding unknown parameters which are possibly correlated can however lead to ambiguous determinations. In addition these parameters remain model-dependent and can lead to an over-interpretation of the retrieved information. At last, the estimation quality of the parameters strongly depends on the noise 
level of the measurements [22].

In the interpretation of SHG polarized responses, the use of an a priori model ultimately fails in giving a general picture of the measured molecular orientational distribution, primarily because there is no possibility to validate this model. In this work, we propose a generic approach capable of encompassing all available orientational information without the need to rely on a specific model for the orientational distribution in the sample. Along the same lines as what has been developed in the past for polarization analysis in poled polymers doped by SHG active molecules [23], we base our approach on order parameters related to symmetry properties of the molecular orientation distribution function in a sample. This general model includes possible geometries that are not of cylindrical symmetry, which was not accessible in the previously developed cone models for collagen. It also gives general information on the shape of the orientational distribution with estimated parameters which are model-independent. We develop this model in a 2D approach that is adapted for the SHG microscopy geometry, and apply it to the quantitative imaging of molecular order parameters in assemblies of isolated collagen fibrils. A numerical model based on Monte Carlo analysis is furthermore implemented to quantify empirically the estimation precision on these parameters, assuming signals limited by Poisson noise.

\section{MOLECULAR DISTRIBUTION}

\section{A. Reduction of the molecular orientational 3D distribution to a 2D distribution}

A polarized Second Harmonic Generation signal from an ensemble of molecules excited in a focal volume originates from the radiation of a macroscopic nonlinear induced dipole $\mathbf{P}^{2 \omega}$, resulting from the orientational average of molecular nonlinear induced dipoles $\mathbf{p}^{2 \omega}$ :

$$
\mathbf{P}^{2 \omega}=N \int_{\Omega} \mathbf{p}^{2 \omega} f(\Omega) d \Omega
$$

with $N$ the molecular density. $f(\Omega)$ defines the molecular angular distribution function with $\Omega=(\theta, \varphi, \psi)$ the Euler angles set defining the molecular orientation. This function models the molecular scale organization of a sample in the macroscopic laboratory frame, and describes the probability to find a molecule oriented along $\Omega$. It is normalized such as $\int_{\Omega} f(\Omega) d \Omega=1$. 
The nonlinear molecular induced dipole originates from the coupling between the molecular nonlinear susceptibility tensor $\beta$ and the incoming fundamental field $\mathbf{E}^{\omega}$ at the $\omega$ frequency:

$$
p_{i}^{2 \omega}=\sum_{j k} \beta_{i j k} E_{j}^{\omega} E_{k}^{\omega}
$$

with $i, j, k$ in the molecular frame $(x, y, z)$.

Writing this dipole in the macroscopic frame $(X, Y, Z)$ and averaging over orientation angles (Eq. 1) results in a macroscopic dipole:

$$
P_{I}^{2 \omega}=\sum_{J K} \chi_{I J K}^{(2)} E_{J}^{\omega} E_{K}^{\omega}
$$

where $\chi_{I J K}^{(2)}$ are the tensorial components of the macroscopic nonlinear susceptibility $\chi^{(2)}$ with $I, J, K$ in the $(X, Y, Z)$ frame (Fig. 1a), and:

$$
\chi_{I J K}^{(2)}=N \int_{\Omega} \beta_{I J K}(\Omega) f(\Omega) d \Omega
$$

where $\beta_{I J K}(\Omega)$ are the molecular susceptibility components expressed in the $(X, Y, Z)$ frame:

$$
\beta_{I J K}(\Omega)=\sum_{i j k} \beta_{i j k}(\mathbf{I} \cdot \mathbf{i})(\mathbf{J} \cdot \mathbf{j})(\mathbf{K} \cdot \mathbf{k})(\Omega)
$$

with $(\mathbf{I} \cdot \mathbf{i})$ the transformation matrix components between the $(x, y, z)$ and the $(X, Y, Z)$ frames.

Note that Eq. 4, which relates molecular scale to macroscopic scale properties, is based on a purely additive model. Accounting for molecule-molecule interactions and molecular packing in dense media $[24,25]$ is often accounted for by a phenomenological tensorial local field correction factor [26], compatible with this additive approach. In what follows, we implicitly embed this correction factor inside the expression of $\chi_{I J K}^{(2)}$, meaning that all molecular order interpretations need to be modulated by the existence of possible local interactions in the medium.

In what follows we consider the case of molecules of one-dimensional symmetry, therefore only $(\theta, \varphi)$ are required to define its orientation (Fig. 1a), and $f(\Omega)$ can be rewritten $f(\theta, \varphi)$. This assumption on the molecular structure is generally made for the investigation of SHG polarized signals from biomolecular assemblies has been shown to be appropriate in collagen [27]. It can be nevertheless generalized to more complex symmetries by introducing 
the appropriate $\beta$ tensor structure. The consequence of multipolar molecular symmetries on the determination of molecular order information is discussed in Section V.

Polarization-resolved SHG consists in rotating the incident fundamental polarization in the sample plane $(X, Y)$, the $Z$ axis defining the propagation direction. Assuming a negligible nonlinear coupling in the $Z$ direction, which is generally the case in samples investigated by nonlinear microscopy imaging $[28,29]$, the only accessible information is restricted to a projection in the $(X, Y) 2 \mathrm{D}$ plane. The projectors $(\mathbf{I} \cdot \mathbf{i})$ with $I=X, Y$ thus only contain terms such as $\sin \theta \cos \varphi$ or $\sin \theta \sin \varphi$ and Eq. 4 can be rewritten in a general form which demonstration is detailed in Appendix A:

$$
\chi_{I J K}^{(2)}=N \int_{0}^{2 \pi} \int_{0}^{\pi} \beta_{I J K}^{*}(\varphi) f(\theta, \varphi) \sin ^{4} \theta d \theta d \varphi
$$

with $\beta_{I J K}^{*}(\varphi)$, defined in Appendix A, encompassing the sum over the microscopic coefficients $\beta_{i j k}$ and the $\varphi$ dependence of its macroscopic projection.

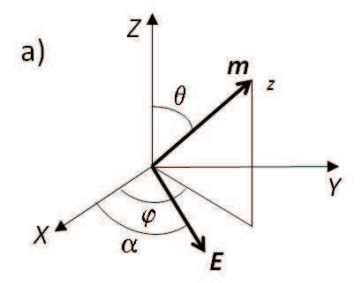

b)
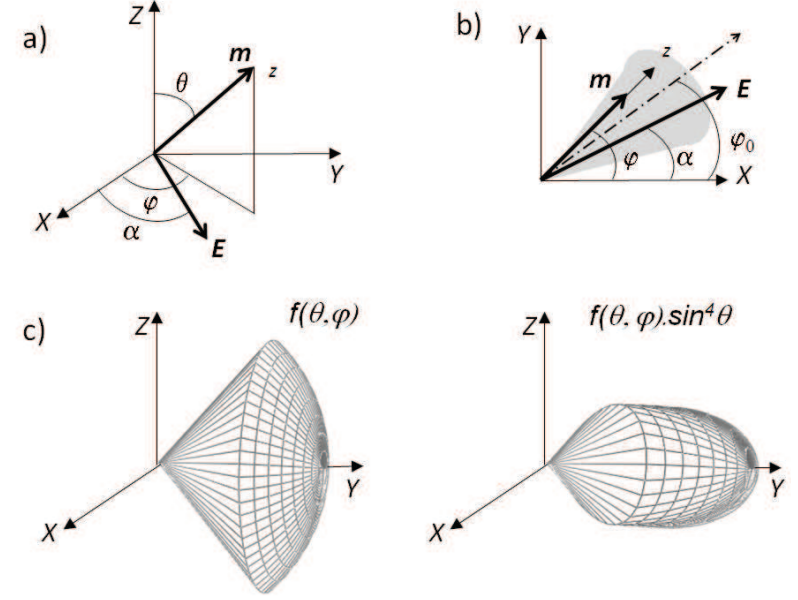

FIG. 1. a) Geometry of the SHG polarized microscopy. $(X, Y)$ is the sample plane, $Z$ is the direction of propagation of the fundamental beam. $\alpha$ is the angle of the incident polarization E relative to $X$. In one-dimensional symmetry molecules, $(\theta, \varphi)$ defines the orientation of the molecular axis $\mathbf{m}$ in the $(X, Y, Z)$ frame. b) Orientation of the $2 \mathrm{D}$ distribution $\left(\varphi_{0}\right)$ represented in a grey shape. $\varphi$ is the orientation of the molecular axis $\mathbf{m}$ relative to $X$ in the sample plane. c) Example of a 3D molecular distribution $f(\theta, \varphi)$ (left) and its modified shape when read by a SHG polarized contrast $f(\theta, \varphi) \sin ^{4} \theta$ (right).

Due to the $2 \mathrm{D}$ constraint of the problem in the plane of polarization, we introduce a new 
distribution function $P(\varphi)$ which only depends on $\varphi$, orientation of the molecule projection in the $(X, Y)$ plane (Fig. 1b):

$$
P(\varphi)=\int_{0}^{\pi} f(\theta, \varphi) \sin ^{4} \theta d \theta
$$

Eq. 7 shows that the relevant distribution function which is effectively read-out in a polarized SHG process is of the form $f(\theta, \varphi) \sin ^{4} \theta$, a function which emphasizes the role of molecules close to the $(X, Y)$ plane compared to out-of plane ones, as depicted in Fig. 1c. In addition, the $2 \mathrm{D}$ distribution $P(\varphi)$ can be used to define the effect of molecular orientation on the polarization resolved SHG signals, using only the molecules orientation information $\varphi$ in the $(X, Y)$ sample plane. $P(\varphi)$ is not a geometrical projection of the $3 \mathrm{D}$ distribution function $f(\theta, \varphi)$ in the $(X, Y)$ frame, but is rather a horizontal sectioning of the 3D molecular distribution imposed by the SHG contrast (Fig. 1c). The macroscopic SHG tensor components in the $(X, Y)$ plane can finally be written:

$$
\chi_{I J K}^{(2)}=N \int_{0}^{2 \pi} \beta_{I J K}^{*}(\varphi) P(\varphi) d \varphi
$$

Thereafter we develop the polarization resolved SHG response of the 2D molecular distribution $P(\varphi)$. In the sample plane, this function can be decomposed on basis of circular functions, appropriate to its 2D geometry:

$$
\begin{aligned}
P(\varphi)= & p_{0}+p_{1} \cos \left(\varphi-\varphi_{0}\right) \\
& +\sum_{n \geq 2} p_{n} \cos \left(n\left(\varphi-\varphi_{0}\right)\right) \\
& +\sum_{n \geq 2} q_{n} \sin \left(n\left(\varphi-\varphi_{0}\right)\right)
\end{aligned}
$$

The $\left(p_{n}, q_{n}\right)$ order terms in Eq. 9 are the signature of different orders of symmetry of the $P(\varphi)$ function, $n=0$ being representative of its isotropic contribution, and even/odd $n$ orders terms being representative of its even/odd orders of symmetry. Moreover, high symmetry orders are representative of more defined angular details. $\varphi_{0}$ is the mean orientation, in the sample plane, of the first order term $n=1$ (Fig. 1b), introduced as a reference orientation angle of the 2D distribution function. Therefore there is no need to introduce a $q_{1}$ parameter. The $q_{n \geq 2}$ terms quantify the orientation of the $n$ order terms with respect to the $X$ axis. Therefore $q_{n \geq 2}$ are also signatures of the absence of a symmetry axis in the distribution function. 
Since SHG, in the dipolar approximation used here, is only sensitive to non-centrosymmetric contributions of a distribution function up to the third order of symmetry [30,31], only the orders 1 and 3 of the distribution function $P(\varphi)$ in Eq. 9 contribute to the SHG response. For this reason, the $2 \mathrm{D}$ orientational distribution function $P(\varphi)$ can be reduced to a truncated $\tilde{P}(\varphi)$ function, which is no longer considered as a probability function:

$$
\begin{aligned}
\tilde{P}(\varphi)= & p_{1} \cos \left(\varphi-\varphi_{0}\right) \\
& +p_{3} \cos \left(3\left(\varphi-\varphi_{0}\right)\right) \\
& +q_{3} \sin \left(3\left(\varphi-\varphi_{0}\right)\right)
\end{aligned}
$$

The information present in $\tilde{P}(\varphi)$ is therefore the relative non-centrosymmetric contribution, of first and third orders, to the complete molecular angular distribution $P(\varphi)$.

\section{B. Single molecule polarized SHG response}

Assuming molecules of one-dimensional symmetry along a $z$ direction in the sample plane (Fig. 1b), $\beta_{i j k}$ possesses a single non vanishing component $\beta_{z z z}$ in the molecular frame, denoted $\beta$ in what follows. In the 2D molecular distribution, the $z$ axis is oriented with an angle $\varphi$ relative to the macroscopic axis $X$. Denoting $\alpha$ the angle of the incident linear polarization $\mathbf{E}$ relative to $X$, the amplitudes of the molecular nonlinear induced dipole along the $X$ and $Y$ axes are therefore proportional to:

$$
\begin{aligned}
& p_{X}^{2 \omega}=E_{0}^{2} \beta \cos \varphi \cos ^{2}(\alpha-\varphi) \\
& p_{Y}^{2 \omega}=E_{0}^{2} \beta \sin \varphi \cos ^{2}(\alpha-\varphi)
\end{aligned}
$$

with $E_{0}$ the amplitude of incoming fundamental field.

The polarization dependence of the total SHG intensity $I(\alpha)$, measured without the use of any analyzer, can be written, in the plane wave approximation, as:

$$
I=I_{X}+I_{Y}=\left|p_{X}^{2 \omega}\right|^{2}+\left|p_{Y}^{2 \omega}\right|^{2}
$$

Therefore:

$$
I(\alpha)=E_{0}^{4} \beta^{2} \cos ^{4}(\alpha-\varphi)
$$

The $\cos ^{4}(\alpha-\varphi)$ function can be decomposed on the basis circular functions $\cos (n(\alpha-\varphi))$ with $n=0,2,4$, which leads to:

$$
I(\alpha)=a_{0}+a_{1} \cos (2(\alpha-\varphi))+a_{2} \cos (4(\alpha-\varphi))
$$


with

$$
\begin{aligned}
& a_{0}=3 E_{0}^{4} \beta^{2} / 8 \\
& a_{1}=E_{0}^{4} \beta^{2} / 2 \\
& a_{2}=E_{0}^{4} \beta^{2} / 8
\end{aligned}
$$

\section{Polarized SHG response in a 2D angular distribution}

The nonlinear response from an ensemble of molecules requires calculating the orientational averaged of single molecule's nonlinear induced dipoles (Eq. 1). The polarization dependence of the total SHG intensity can then be written, in the plane wave approximation, as a quantity proportional to the macroscopic expression $\left|P_{X}^{2 \omega}\right|^{2}+\left|P_{Y}^{2 \omega}\right|^{2}$. Introducing $\tilde{P}(\varphi)$ in Eq. 8, and using the methodology developed in Appendix B, it can be shown that similarly as the single molecule case, this function is decomposed on the basis circular functions $\cos \left(n\left(\alpha-\varphi_{0}\right)\right)$ and $\sin \left(n\left(\alpha-\varphi_{0}\right)\right)$ with $n=0,2,4$ :

$$
\begin{aligned}
I(\alpha)= & a_{0}+a_{1} \cos \left(2\left(\alpha-\varphi_{0}\right)\right)+a_{2} \cos \left(4\left(\alpha-\varphi_{0}\right)\right) \\
& +b_{1} \sin \left(2\left(\alpha-\varphi_{0}\right)\right)+b_{2} \sin \left(4\left(\alpha-\varphi_{0}\right)\right)
\end{aligned}
$$

with

$$
\begin{array}{ll}
a_{0}=\frac{A}{16}\left(5 p_{1}^{2}+p_{3}^{2}+q_{3}^{2}\right) & \\
a_{1}=\frac{A}{4}\left(p_{1}^{2}+p_{1} p_{3}\right) & a_{2}=\frac{A}{8} p_{1} p_{3} \\
b_{1}=\frac{A}{4} p_{1} q_{3} & b_{2}=\frac{A}{8} p_{1} q_{3}
\end{array}
$$

where $A=N^{2} E_{0}^{4} \pi^{2} \beta^{2}$.

This development shows that the total SHG intensity can be decomposed in a simple Fourier decomposition, in a similar manner as already introduced in previous works [22, 32],

with coefficients depending on the orders of the $\tilde{P}(\varphi)$ function. Due to the coherent nature of the SHG process, this dependence is however not a pure linear combination of the $p_{1}, p_{3}, q_{3}$ orders, but rather a nonlinear mixing which prevents the simple resolution of an inverse problem. In the most general situation, all the orders can be however determined as a unique solution, as long as the first order $p_{1}$ does not vanish. Indeed in the case of a pure third order distribution $\left(p_{1}=0\right)$, only $a_{0}$ does not vanish and the total intensity will be a constant function of $\alpha$, the distribution function exhibiting in this case a $2 \pi / 3$ invariant 
rotation while the fundamental electromagnetic field exhibits a $\pi$ invariant rotation, making impossible any signal modulation. This property has been previously exploited in octupolar molecular media to obtain polarization-independent nonlinear responses [30, 33].

\section{ESTIMATION OF ORDER PARAMETERS}

\section{A. Principle of the estimation of the order parameters}

As mentioned above, the non linear expression of the Fourier coefficients $a_{0}, a_{1}, a_{2}, b_{1}, b_{2}$ as functions of the unknown parameters $A, p_{1}, p_{3}, q_{3}$ makes impossible an analytical resolution of the inverse problem. In a polarization resolved measurement, $A$ is not relevant since it is a pure magnitude factor. The measurement being not absolute, a normalization is necessary and for this reason we chose to estimate three remaining parameters: the ratios $p=p_{3} / p_{1}$, $q=q_{3} / p_{1}$ (called "order parameters" in what follows), and $\varphi_{0}$. The distribution function becomes therefore a more simple expression:

$$
\begin{aligned}
\tilde{P}(\varphi)= & \cos \left(\varphi-\varphi_{0}\right)+p \cos \left(3\left(\varphi-\varphi_{0}\right)\right) \\
& +q \sin \left(3\left(\varphi-\varphi_{0}\right)\right)
\end{aligned}
$$

The SHG intensity $I(\alpha)$, which depends on the mean orientation $\varphi_{0}$ and the $(p, q)$ parameters, will be written $I\left(w(p, q), \varphi_{0}, \alpha\right)$ with $w(p, q)=\left(a_{0}, a_{1}, a_{2}, b_{1}, b_{2}\right)$, the measured Fourier coefficients of Eq. 16, which depend only on $p$ and $q$. A typical experimental measurement leads to intensity values $I_{\exp }\left(\alpha_{i}\right)$ for $m$ regularly spaced measurement angles $\alpha_{i}$ with $i=1 . . m$, with $m$ high enough to allow the unambiguous determination of the Fourier coefficients of Eq. 16. To retrieve the $\varphi_{0}$ and $(p, q)$ information, a minimization can be performed of the mean square error between the measured intensity $I_{\text {exp }}\left(\alpha_{i}\right)$ and the model intensity $I\left(w(p, q), \varphi_{0}, \alpha_{i}\right)$. In practice, we implement a minimization procedure on the Fourier coefficients of the intensities. A minimization is separately performed on the $\varphi_{0}$ parameter since it appears as a pure phase parameter in Eq. 16. For each $\varphi_{0}$, the estimation operation consists in finding the couple $(p, q)$ which minimizes the error function:

$$
E\left(w(p, q), \varphi_{0}\right)=\left\|w_{e x p}\left(\varphi_{0}\right)-w(p, q)\right\|^{2}
$$

where $w_{\text {exp }}\left(\varphi_{0}\right)$ is the set of Fourier coefficients measured on the experimental SHG intensity artificially rotated by a $\varphi_{0}$ angle. 


\section{B. Experimental setup}

The nonlinear polarimetric microscope used in this work has been described in previous works [28]. The excitation light source is a tunable Ti:Sapphire laser that delivers 150 fs pulses at a repetition rate $80 \mathrm{MHz}$. The incident wavelength is set at $800 \mathrm{~nm}$ for SHG with a typical averaged power of a few $\mathrm{mWs}$ at the sample plane. The laser beam is reflected by a dichroic mirror and focused on the sample by a high Numerical Aperture (NA) objective ( $\times 40$, NA 1.15). The backward emitted signal is collected by the same objective and directed to an avalanche photodiode. The total intensity is collected without any analyzer. Previous works have shown that using no analyzer can lead to misleading interpretation in case of polarization distortions by the sample [34], therefore in the present case we preliminary verify that the sample does not exhibit such distortions following the methodology developed in [34]. Images are performed by scanning the sample with two galvanometric mirrors. The linear polarization of the incident laser beam is continuously rotated in the sample plane by an achromatic half wave plate mounted on a step rotation motor at the entrance of the microscope. For each value $\alpha_{i}$ of the polarization angle relative to $X$ (in total 32 angles between $0^{\circ}$ and $180^{\circ}$ ), a SHG image is recorded. At last, polarization distortions (dichroism and ellipticity) originating from the mirrors reflection are preliminarily characterized following a calibration procedure described previously [28]. The ellipticity is found to be close to $20^{\circ}$ (with a dichroism factor close to 0 ), which is seen to induce a negligible bias in the estimation of the $p$ and $q$ parameters. At last, the polarization mixing effects induced by the high numerical aperture at both excitation and collection steps can be ignored in a first approximation for nonlinear dipoles principally lying close to the sample plane $[28,29]$, therefore the planar approximation used above to calculate the SHG intensity radiation can be considered as valid.

\section{Results}

As an illustration of the technique, polarization resolved SHG experiments have been performed on fibrils of collagen type I of submicrometric diameters, coated on a glass substrate following a procedure already established [35]. The choice of this biological model is motivated by the fact that the fibrils are quite isolated from each other and not embed- 
ded in a dense tissue, which might distort polarization properties by scattering or birefringence $[34,36]$. The structure of collagen type I fibrils is furthermore well known and has been studied previously by polarization resolved SHG imaging [19-21].

The usually proposed model supposes that collagen fibrils or larger fibrils are made of SHG active one-dimensional molecules oriented along a triple helix structure of given pitch angle and period [19], which confers to them an averaged orientation distribution function lying along a cone surface. In this work we only assume that molecules are of one-dimensional geometry, without any further hypothesis on the orientational model. This allows in particular to address the question of distribution functions distortions in fibrils assemblies which are not necessary of cylindrical symmetry. At last, the macroscopic orientation of single fibrils can be directly visualized from the SHG image, which allows direct comparison with the obtained estimated orientation parameter for $\varphi_{0}$.

A typical SHG image from collagen fibrils depicted in Fig. 2a shows elongated fibrils of various diameters and lengths, averaged intensities and orientations. For each pixel of this image, the mean orientation of the molecular distribution $\varphi_{0}$, as well as the $p$ and $q$ order parameters are estimated by minimization of the $E\left(w(p, q), \varphi_{0}\right)$ estimator introduced in Eq. 19, using sampling values of $10^{\circ}$ between $0^{\circ}$ and $180^{\circ}$ for $\varphi_{0}$, and 0.02 between -3 and 3 for $(p, q)$. The chosen range values for $(p, q)$ is specific to collagen (see section $\mathrm{V}$ ), and can be adapted for other type of samples. As can be observed in Fig. $2 b$, the obtained $\varphi_{0}$ values are in good agreement with the macroscopic orientation of the fibrils in the sample plane, supporting the fact that molecules orient on average along a main fibril direction (note that $\varphi_{0}=0^{\circ}$ and $\varphi_{0}=180^{\circ}$ are equivalent angles, therefore leading to a mixture of these two values in the image of horizontal fibrils). The $p$ and $q$ order parameters images are represented in Fig. 2c,d, and also as histograms in Fig. 2e,f. Note that we chose to represent the images (Fig. 2a-d) with a threshold of 60 photons/pixel (SHG signal summed over all 32 incident polarizations) in order to depict the large population of fibrils and intensities available in this sample. The threshold intensity chosen to represent the histograms (150 photons/pixel) is higher since a high signal to noise ratio is required to analyze the data with sufficient precision (see Section IV).

The averaged value of $p$ obtained over the whole image is -0.47 , which means that the first and third order contributions to the angular distribution function $\tilde{P}(\varphi)$ are pointing 

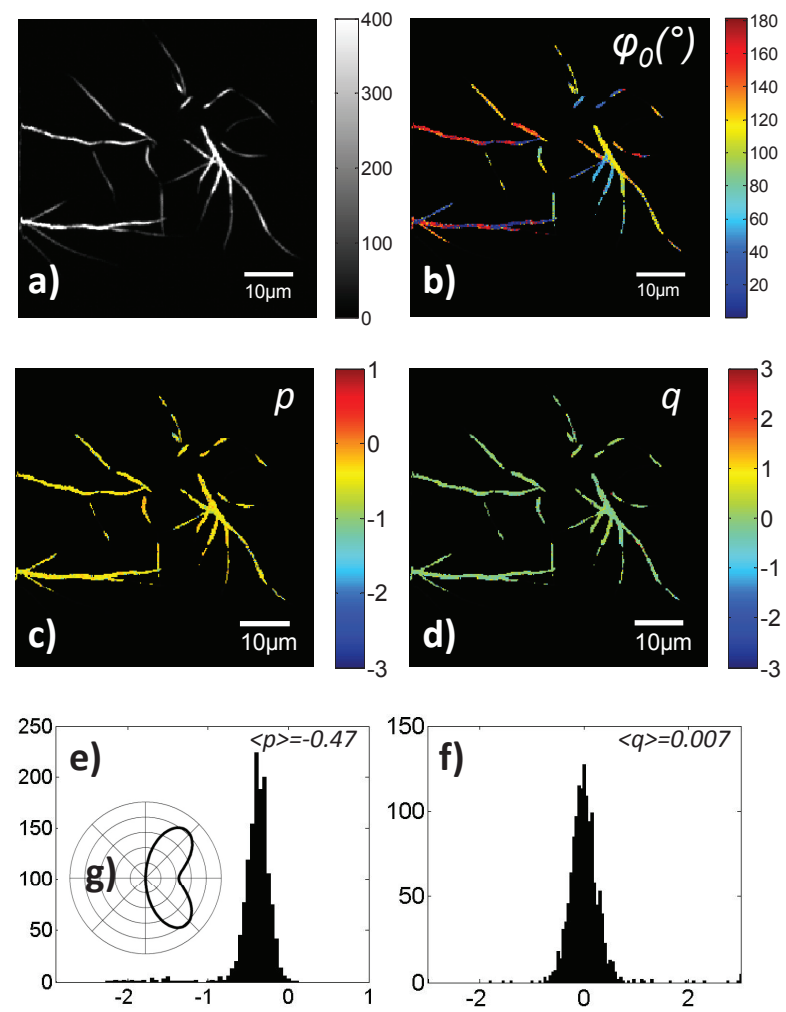

FIG. 2. a) SHG Intensity image of isolated Collagen type I fibrils coated on the sample plane surface. The intensity scale is the sum of SHG signals over the 32 incident polarizations (threshold: 60 photons/pixel). b-d) Images of the parameters obtained from a Fourier decomposition of the intensity polarization dependence: b) $\varphi_{0}$ (degrees); c) $p$; d) $q$. e) histogram of $p$ on the whole image; f) histogram of $q$ on the whole image. For the histograms the threshold of the analyzed pixels is 150 photons/pixel. g) polar representation of the truncated distribution function $\tilde{P}(\varphi)$ deduced from the averaged $p$ and $q$ values obtained over the whole image.

towards opposite directions. This value leads to a depression in the truncated distribution in its center, represented in Fig. $2 \mathrm{~g}$, meaning that there are more SHG-active molecules on the side of the distribution than in its center. The two-lobes of this distribution are separated by an angular aperture of about $50^{\circ}$, which is also close to the cone half aperture angle found in previous works on dense collagen structures, assuming a cone surface shape [19, 21]. This result provides a direct evidence that the traditionally assumed distribution shape of cone surface can be considered as valid in ordered collagen structures, which would be otherwise impossible to verify. Third, the averaged value of $q$ close to 0 means that the first and 
third order contributions functions exhibit the same averaged orientation, supporting the fact that the global angular distribution function exhibits an axis of symmetry (in $3 \mathrm{D}$, this would correspond to a cylindrical symmetry).

In order to visualize the distribution function obtained locally in collagen fibrils, a small image section is selected and represented in Fig. 3a. This image contains different populations : isolated collagen fibrils of different orientations, and an overlap between fibrils. In the regions where collagen fibrils are clearly isolated, the truncated distribution function $\tilde{P}(\varphi)$ is globally oriented in the direction of the fibrils, with a two-lobes shape resembling the averaged distribution function (Fig. 3c). In a region where the two fibrils intersect, the distribution function shows more pronounced fine lobes at a larger aperture angle. This distribution could be interpreted as the result of a higher disorder, although it is not possible to discriminate between a molecular origin for this disorder (change of chemical composition and helix structures in collagen) and a microscopic scale disorder made of a different fibrils orientations. Nevertheless this example illustrates the potential of this method to provide a direct visualization of the local disorder in a collagen fibrils sample. Using a pure cone surface model as usually inferred would not be able to provide this level of information, since it is not sensitive to pure distribution shape changes.

\section{ESTIMATION ACCURACY}

\section{A. Monte-Carlo simulations}

The accuracy of the estimation of the order parameters is studied assuming that measurements are limited by Poisson noise, which is verified in the present case. To quantify the accuracy of the method, Monte-Carlo experiments have been performed. A polarization resolved signal is created using Eqs. 16 and 17, for a chosen set of parameters $\varphi_{0}, p_{1}, p_{3}$ and $q_{3}$, and furthermore introducing Poisson noise to the signal. The values of the parameters have been chosen to be close to the averaged ones found experimentally. From this signal, the parameters $\varphi_{0}$ and $(p, q)$ are then estimated following the same methodology as previously described on experimental data. 2000 realizations are executed for several levels of noise (varying the SHG total intensity from 10 photons to 160 photons, summed over all 32 incident polarization angles). For each level of noise the mean value and the variance of the 


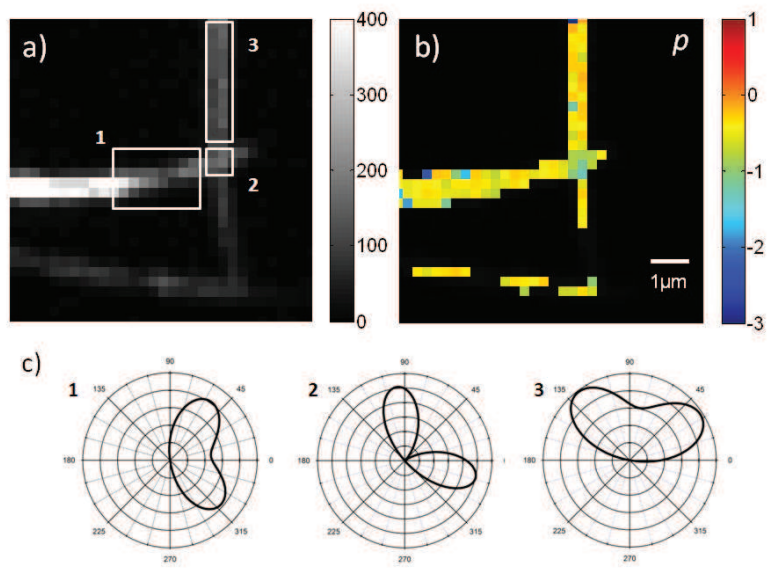

FIG. 3. a) SHG image of a selected region of Fig. 2. b) Corresponding image of $p$. c) Polar representation of the averaged distribution function $\tilde{P}(\varphi)$ obtained from the retrieved $\left(\varphi_{0}, p, q\right)$ parameters on three regions of interest represented as white rectangles in the SHG image. These functions are obtained using the average of the retrieved parameter within the region of interest (only active pixels are used): $1\left(p=-0.4, q=0.04, \varphi_{0}=8^{\circ}\right) ; 2\left(p=-0.98, q=0.04, \varphi_{0}=42^{\circ}\right)$; and $3\left(p=-0.38, q=0.01, \varphi_{0}=80^{\circ}\right)$. The error of estimation on the parameters produces an error bar on the angular distribution which is smaller than the thickness of the line used to draw the distribution function, therefore it is not represented here.

$\varphi_{0}$ and $(p, q)$ parameters are calculated. These results are reported in Fig. 4. As expected, the variances of the estimated parameters are seen to decrease linearly with the number of photons. Moreover, stronger variances values are observed for the $q$ parameter. This can be due to the fact that the determination of $q$ relies on a set of equations (Eq. 17) which contains some redundancies, contrary to $p$. Overall this result provides indication on the level of signal to reach in order to estimate the order parameters with a reasonable quality. It is for instance visible that the total number of photon should be above 150 to reach a good estimation precision with variances below 0.05 , for all parameters in the situation considered here.

\section{B. Experimental accuracy}

To give a quantitative picture of the experimental accuracy and compare it to predictions from Monte Carlo simulations, seven experiments were performed on a same region 
of the collagen type I fibrils sample, using identical experimental conditions. A fibril with orientation $\varphi_{0} \sim 45^{\circ}$, lying in the middle of Fig. 2, is chosen to evaluate the $\left(\varphi_{0}, p, q\right)$ parameters for each of its pixels (in total 78 pixels are analyzed) and for each experiment. The variance of these parameters is calculated for each pixel based on their seven estimated values. This procedure allows a direct calculation of the parameters' variance, avoiding any dependence on possible sample heterogeneities within the same collagen fiber. For each pixel, the variance of the $\varphi_{0}$ and $(p, q)$ parameters is determined as well as the corresponding total number of photons, defined as the average of the total number of photons measured over the seven experiments. Although this procedure does not permit to explore a large range of total number of photons and is based on a small number of measurements, it gives a rough idea of the experimental accuracy of the parameters. To represent the global behavior of a given parameter in Fig. 4, the variances obtained for all 78 measured pixels are grouped by intensity ranges (of [0-10] photons, [10-20] photons, etc.) and averaged for each range. The obtained variances are seen to lie globally close to values expected from the Monte-Carlo simulation, which emphasizes the role of Poisson noise in our measurement.

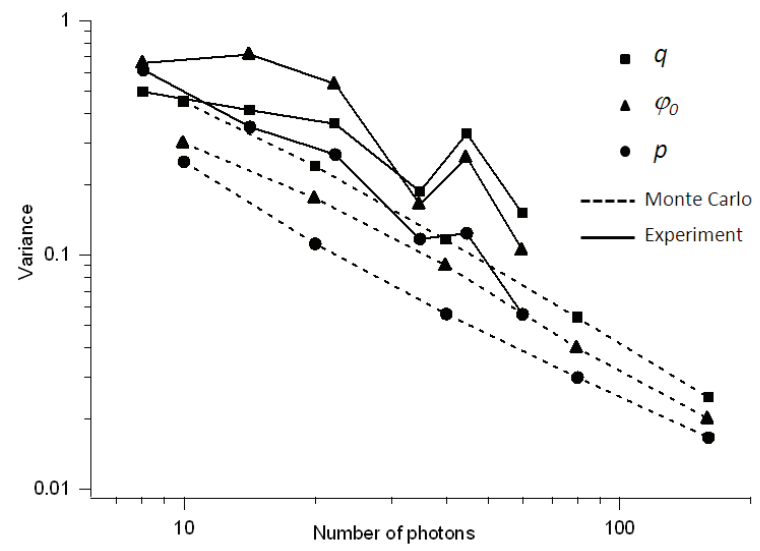

FIG. 4. Variances of the $p, q$ and $\varphi_{0}$ parameters as functions of the total number of photon (summed over the 32 incident polarizations). Circles: $p$, squares: $q$, triangles: $\varphi_{0}$. Dashed lines: Monte-Carlo simulations using the initial parameters $p=-0.4, q=0, \varphi_{0}=45^{\circ}$. Continuous lines: experimental variances measured over seven experiments performed for a same region of interest, on a fibril of orientation $\varphi_{0} \sim 45^{\circ}$ relative to $X$. Each point represents the average of the variances obtained in a range of photon number: [0-10] photons, [10-20] photons, etc. 


\section{INTERPRETATION OF THE ORDER PARAMETERS}

It is possible to relate the obtained $p$ and $q$ parameters to the models used previously in the literature, using their definition in terms of Fourier decomposition coefficients of the molecular distribution.

\section{A. Relation between order parameters and an explicit molecular distribution func-} tion

In a 2D molecular orientational distribution model where $P(\varphi)$ is a priori assumed, the $p_{n}$ and $q_{n}$ parameters of the Fourier decomposition of $P(\varphi)$ are defined as:

$$
\begin{aligned}
& p_{n}=\int_{0}^{2 \pi} P(\varphi) \cos (n \varphi) d \varphi \\
& q_{n}=\int_{0}^{2 \pi} P(\varphi) \sin (n \varphi) d \varphi
\end{aligned}
$$

We first consider the case of a $f(\theta, \varphi)$ distribution made of a 3D cone filled with molecules with a total angular aperture $2 \psi_{0}$, lying in the $(X, Y)$ plane along the $X$ axis (we choose here $\varphi_{0}=0$ for simplification). In this situation, the corresponding $2 \mathrm{D}$ function $P(\varphi)$ can be approximated by:

$$
P(\varphi)=\frac{1}{2 \psi_{0}} \operatorname{Rect}\left[\varphi,-\psi_{0}, \psi_{0}\right]
$$

with the Rect function defines as 1 for $-\psi_{0} \leq \varphi \leq \psi_{0}$ and 0 elsewhere. This leads to:

$$
p_{n}=\frac{\sin \left(n \psi_{0}\right)}{\pi n \psi_{0}} ; q_{n}=0
$$

When $\psi_{0} \rightarrow 0$ (small cone apertures) then $p_{n} \rightarrow 1 / \pi$, and when $\psi_{0} \rightarrow \pi / 2$ (large apertures) then $p_{2 n+1} \rightarrow \frac{(-1)^{n}}{(2 n+1) \pi^{2} / 2}$, therefore $p=p_{3} / p_{1} \rightarrow-1 / 3$, which fixes a lower limit of the measurable $p$ parameter in this case.

We then consider the case of a cone surface distribution where the molecules only lie on the surface of a cone of angular aperture $2 \psi_{0}$. This situation is specifically used to explore molecular order in collagen based samples. In this case:

$$
P(\varphi) \simeq \frac{1}{2}\left(\delta\left[\varphi-\psi_{0}\right]+\delta\left[\varphi+\psi_{0}\right]\right)
$$

which leads to:

$$
p_{n}=\frac{1}{\pi} \cos \left(n \psi_{0}\right) ; q_{n}=0
$$


When $\psi_{0} \rightarrow 0$ then $p_{n} \rightarrow 1 / \pi$, and when $\psi_{0} \rightarrow \pi / 2$ then $p_{2 n+1} \rightarrow 0$. Furthermore, for $\psi_{0}=\pi / 2-\epsilon$, then $p_{2 n+1} \simeq \frac{1}{\pi}(-1)^{n}(2 n+1) \epsilon$ and thus $p=p_{3} / p_{1} \simeq-3$. In this case, the minimum expected value for $p$ is therefore lower than for a filled cone distribution.

The dependencies of $p$ as a function of the cone half aperture $\psi_{0}$ are represented in Fig. 5a for the two models, filled cone and cone surface. From this figure one can draw several observations. First, using $p$ to determine $\psi_{0}$ can lead to different solutions depending on the model used, since the interpretation of $\psi_{0}$ is model-dependent. In the present approach, a cylindrical symmetry distribution is defined by one unique order parameter $p$, which is not model-dependent and is representative of the accessible quantity from polarization dependent SHG. Second, one can draw additional conclusions on the shape of the distribution, based on the measurement of $p$ and $q$. Indeed in the case where $p$ takes large negative values (typically $p<-1 / 3$ ), the 2D distribution function exhibits angularly a depression in its center, which makes it resemble more a surface cone distribution than a filled cone. This is illustrated in the polar representation of the $\tilde{P}(\varphi)$ distribution (Fig. 5b). Note that any kind of hollow distribution in its center would lead to a similar behavior as observed for the cone surface.

In the case of investigations on collagen, models of cone surfaces have been extensively used and typical values found in the literature lie close to $\psi_{0} \sim 45-60^{\circ}[17,21]$, leading to $p$ ranging from -0.3 to -1.4 , which is the range found in single isolated fibrils in our measurements. The determination of $p$ and $q$ provides however here extra information which is not accessible in approaches based on a priori supposed models, in particular by revealing explicitly the hollow shape distribution and possible deviations from a cylindrical symmetry.

Finally, note that this whole derivation is based on the initial assumption that the angular distribution is made of pure 1D molecules exhibiting a unique diagonal $\beta_{z z z}$ coefficient, which has been shown to be robust in the case of collagen studies. Introducing a more complex molecular symmetry in Eq. 5 would lead to a deviation of the $p$ values deduced here, which can be strong if significant off-diagonal molecular nonlinear coefficients arise. Along the same line as previously developed approaches $[16,17,19-21]$, the estimation of $p$ therefore relies on either an assumed or an a priori known molecular structure. 


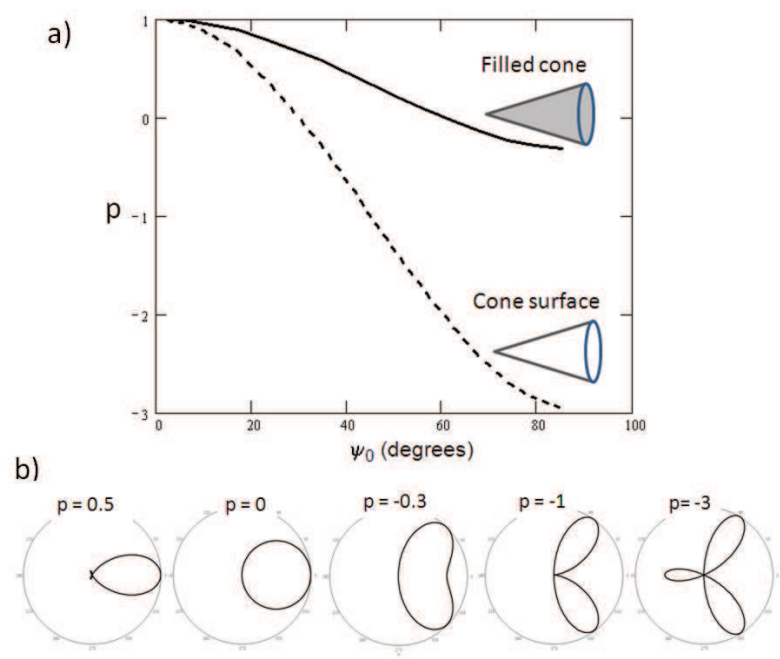

FIG. 5. a) Dependency of the $p$ parameters as a function of the cone half aperture $\psi_{0}$ for two models of the molecular 2D distribution function $\tilde{P}(\varphi)$ : filled cone (continuous line), cone surface (dashed line). b) Different distributions $\tilde{P}(\varphi)$ represented as polar plots in the sample plane, for $p=0.5,0,-1 / 3,-1,-3$.

\section{B. Relation between order parameters and microscopic tensorial coefficients}

The structural parameters of molecular assemblies are often expressed in terms of their microscopic tensorial nonlinear coefficients, which relate the molecular order information to a crystallographic point of view. In the case of collagen, a $C_{6}$ symmetry is assumed from the known crystalline structure of this macromolecule [3]. Denoting 3 the high symmetry axis of the $C_{6}$ symmetry cylinder and $(1,2)$ its perpendicular plane, the collagen symmetry is therefore reduced to two microscopic tensorial coefficients under non-resonant excitation conditions: $\chi_{333}^{(2)}$ and $\chi_{311}^{(2)}=\chi_{322}^{(2)}$ (with all index permutation permitted in case of valid Kleinman symmetry conditions). The ratio $\chi_{333}^{(2)} / \chi_{311}^{(2)}$, often used to characterize molecular order in collagen, has been found to range between 0.8 and 2.6 [3, 16, 17, 19-21]. A relation between $\chi_{333}^{(2)} / \chi_{311}^{(2)}$ and the aperture angle $\psi_{0}$ of the equivalent cone surface model mentioned above can be deduced from tensorial distribution expression similar to Eq. 4, leading to [21]:

$$
\tan ^{2}\left(\psi_{0}\right)=2 \frac{\chi_{311}^{(2)}}{\chi_{333}^{(2)}}
$$

In the present analysis, the value found for $p=-0.4$ in isolated fibrils leads to $\chi_{333}^{(2)} / \chi_{311}^{(2)}=$ 1.85, which lies in the range of values previously found in tissues. 


\section{CONCLUSION}

We have introduced a generic approach for the analysis of polarization dependent SHG microscopy measurement, applied to molecular and biological structural imaging. This approach is based on a $2 \mathrm{D}$ derivation of the problem, accounting for the fact that the read-out of the polarization information in the sample plane necessarily reduces the problem to the investigation of an angular distribution function lying in this plane. We derived a simple way to retrieve molecular order parameters from a Fourier decomposition of the SHG polarization responses, and showed their estimation precision as a function of the signal level, based on a Poisson noise statistics. The obtained parameters, based on a circular decomposition of the molecular distribution function, are shown to contain richer information than the traditional model-oriented estimations. We illustrated the application of this new approach for the imaging of molecular order in collagen type I fibrils, where order information can be obtained at the microscopic scale without the need to infer a specific orientational model.

\section{ACKNOWLEDGMENTS}

We wish to acknowledge Philippe Réfrégier for his key contribution to the discussions. We also thank Marie Guilbert and Pierre Jeannesson (Université Reims Champagne Ardennes) for the preparation of the collagen type I fibrils sample. We thank the Provence Alpes Côte d'Azur (PACA) Region and CNRS for financial support.

\section{Appendix A: From 3D to 2D expressions of the nonlinear SHG macroscopic re-} sponse

The expression of the macroscopic nonlinear SHG response is based on calculation of the $\beta_{I J K}(\Omega)$ molecular components expressed in the macroscopic frame $(X, Y, Z)$. Assuming molecules of one-dimensional symmetry along a $z$ direction in the sample plane, $\beta_{i j k}$ possesses a single non vanishing component $\beta_{z z z}($ denoted $\beta)$ in the molecular frame. We denote $(\theta, \varphi)$ the orientation of the $z$ axis in the $(X, Y, Z)$ frame. In the case the incident polarization lies in the $(X, Y)$ plane, only four macroscopic coefficients need to be considered (index 
permutation are allowed):

$$
\begin{aligned}
& \beta_{X X X}(\theta, \varphi)=\beta \sin ^{3} \theta \cos ^{3} \varphi \\
& \beta_{X Y Y}(\theta, \varphi)=\beta \sin ^{3} \theta \cos \varphi \sin ^{2} \varphi \\
& \beta_{Y Y Y}(\theta, \varphi)=\beta \sin ^{3} \theta \sin ^{3} \varphi \\
& \beta_{Y X X}(\theta, \varphi)=\beta \sin ^{3} \theta \cos ^{2} \varphi \sin \varphi
\end{aligned}
$$

Therefore that the $\beta_{I J K}$ coefficients (with $\left.(I, J, K)=(X, Y)\right)$ can be written in a general way as:

$$
\beta_{I J K}(\theta, \varphi)=\beta_{I J K}^{*}(\varphi) \sin ^{3} \theta
$$

with $\beta_{I J K}^{*}(\varphi)$ encompassing all $\varphi$ dependence sinusoidal functions. Finally the macroscopic nonlinear tensorial coefficients can be written:

$$
\begin{aligned}
\chi_{I J K=(X, Y)}^{(2)} & =N \int_{0}^{2 \pi} \int_{0}^{\pi} \beta_{I J K} f(\theta, \varphi) \sin \theta d \theta d \varphi \\
& =N \int_{0}^{2 \pi} \int_{0}^{\pi} \beta_{I J K}^{*}(\varphi) f(\theta, \varphi) \sin ^{4} \theta d \theta d \varphi
\end{aligned}
$$

\section{Appendix B: SHG response from an ensemble of molecules}

In this part we derive the expression of the Fourier coefficients of the SHG intensity polarization dependence for an ensemble of molecules. We consider a one-dimensional planar molecule lying in the sample plane, its main nonlinear coefficient $\beta_{z z z}$ being denoted $\beta$. The $z$ axis is oriented with an angle $\varphi$ relative to the macroscopic axis $X$. $\alpha$ the angle of the incident linear polarization $\mathbf{E}$ relative to $X$. The amplitudes of the molecular nonlinear induced dipole along the $X$ and $Y$ axes are proportional to:

$$
\begin{aligned}
& p_{X}^{2 \omega}=E_{0}^{2} \beta \cos \varphi \cos ^{2}(\alpha-\varphi) \\
& p_{Y}^{2 \omega}=E_{0}^{2} \beta \sin \varphi \cos ^{2}(\alpha-\varphi)
\end{aligned}
$$

with $E_{0}$ the amplitude of incoming fundamental field.

Using trigonometric formula, these expressions can be written as:

$$
\begin{aligned}
& p_{X}^{2 \omega}=\mathcal{A}_{0}+\mathcal{A}_{c} \cos (2 \alpha)+\mathcal{A}_{s} \sin (2 \alpha) \\
& p_{Y}^{2 \omega}=\mathcal{B}_{0}+\mathcal{B}_{c} \cos (2 \alpha)+\mathcal{B}_{s} \sin (2 \alpha)
\end{aligned}
$$


where

$$
\begin{aligned}
& \mathcal{A}_{0}=u \cos \varphi \\
& \mathcal{B}_{0}=u \sin \varphi \\
& 2 \mathcal{A}_{c}=u(\cos \varphi+\cos (3 \varphi)) \\
& 2 \mathcal{A}_{s}=u(\sin \varphi+\sin (3 \varphi)) \\
& 2 \mathcal{B}_{c}=u(-\sin \varphi+\sin (3 \varphi)) \\
& 2 \mathcal{B}_{s}=u(\cos \varphi-\cos (3 \varphi))
\end{aligned}
$$

with $u=E_{0}^{2} \beta / 2$

In the case of a distribution made of an ensemble of molecules, we introduce the expansion of the molecular distribution on the basis of circular functions:

$$
\begin{aligned}
P(\varphi)= & p_{0}+p_{1} \cos \left(\varphi-\varphi_{0}\right)+\sum_{n \geq 2} p_{n} \cos \left(n \varphi-n \varphi_{0}\right) \\
& +\sum_{n \geq 2} q_{n} \sin \left(n \varphi-n \varphi_{0}\right)
\end{aligned}
$$

The coherent SHG intensity from this molecular distribution is deduced from its macroscopic nonlinear induced dipole, defined as the orientational average of the molecular nonlinear induced dipole:

$$
I=\left|N\left\langle p_{X}^{2 \omega}\right\rangle\right|^{2}+\left|N\left\langle p_{Y}^{2 \omega}\right\rangle\right|^{2}
$$

with $N$ the molecular density and $\langle\ldots\rangle$ the orientational averaging operation defined as:

$$
\langle A(\varphi)\rangle=\int_{0}^{2 \pi} A(\varphi) P(\varphi) d \varphi
$$

This orientation averaging involves moments of the molecular distribution:

$$
\langle\cos (m \varphi)\rangle=\int_{0}^{2 \pi} \cos (m \varphi) P(\varphi) d \varphi
$$

which can further be written as:

$$
\langle\cos (m \varphi)\rangle=p_{m} \pi \cos \left(m \varphi_{0}\right)-q_{m} \pi \sin \left(m \varphi_{0}\right)
$$

The same calculus is made for the sin moment:

$$
\langle\sin (m \varphi)\rangle=p_{m} \pi \sin \left(m \varphi_{0}\right)+q_{m} \pi \cos \left(m \varphi_{0}\right)
$$

The different moments necessary for the calculation of the macroscopic nonlinear dipole are simply:

$$
\begin{aligned}
\langle\cos (\varphi)\rangle & =p_{1} \pi \cos \left(\varphi_{0}\right) \\
\langle\sin (\varphi)\rangle & =p_{1} \pi \sin \left(\varphi_{0}\right) \\
\langle\cos (3 \varphi)\rangle & =p_{3} \pi \cos \left(3 \varphi_{0}\right)-q_{3} \pi \sin \left(3 \varphi_{0}\right) \\
\langle\sin (3 \varphi)\rangle & =p_{3} \pi \sin \left(3 \varphi_{0}\right)+q_{3} \pi \cos \left(3 \varphi_{0}\right)
\end{aligned}
$$


Therefore:

$$
\begin{aligned}
\left\langle\mathcal{A}_{0}\right\rangle= & u p_{1} \pi \cos \varphi_{0} \\
\left\langle\mathcal{B}_{0}\right\rangle= & u p_{1} \pi \sin \varphi_{0} \\
\frac{2}{\pi}\left\langle\mathcal{A}_{c}\right\rangle= & u\left[p_{1} \cos \varphi_{0}\right. \\
& \left.+p_{3} \cos \left(3 \varphi_{0}\right)-q_{3} \sin \left(3 \varphi_{0}\right)\right] \\
\frac{2}{\pi}\left\langle\mathcal{A}_{s}\right\rangle= & u\left[p_{1} \sin \varphi_{0}\right. \\
& \left.+p_{3} \sin \left(3 \varphi_{0}\right)+q_{3} \cos \left(3 \varphi_{0}\right)\right] \\
\frac{2}{\pi}\left\langle\mathcal{B}_{c}\right\rangle= & u\left[-p_{1} \sin \varphi_{0}\right. \\
& \left.+p_{3} \sin \left(3 \varphi_{0}\right)+q_{3} \cos \left(3 \varphi_{0}\right)\right] \\
\frac{2}{\pi}\left\langle\mathcal{B}_{s}\right\rangle= & u\left[p_{1} \cos \varphi_{0}\right. \\
& \left.-p_{3} \cos \left(3 \varphi_{0}\right)+q_{3} \sin \left(3 \varphi_{0}\right)\right]
\end{aligned}
$$

The SHG intensity can finally be written as:

$$
\begin{aligned}
I(\alpha)= & a_{0}+a_{1} \cos \left(2\left(\alpha-\varphi_{0}\right)\right)+a_{2} \cos \left(4\left(\alpha-\varphi_{0}\right)\right) \\
& +b_{1} \sin \left(2\left(\alpha-\varphi_{0}\right)\right)+b_{2} \sin \left(4\left(\alpha-\varphi_{0}\right)\right)
\end{aligned}
$$

with:

$$
\begin{array}{ll}
a_{0}=\frac{A}{16}\left(5 p_{1}^{2}+p_{3}^{2}+q_{3}^{2}\right) & \\
a_{1}=\frac{A}{4}\left(p_{1}^{2}+p_{1} p_{3}\right) & a_{2}=\frac{A}{8} p_{1} p_{3} \\
b_{1}=\frac{A}{4} p_{1} q_{3} & b_{2}=\frac{A}{8} p_{1} q_{3}
\end{array}
$$

where $A=N^{2} E_{0}^{4} \pi^{2} \beta^{2}$.

[1] J. Gannaway, and C.J.R. Sheppard, "Second harmonic imaging in the scanning optical microscope," Opt. and Quant. Elec. 10, 435-439 (1978).

[2] M. Flörsheimer, C. Radüge, H. Salmen, M. Bösch, R. Terbrack, and H. Fuchs, "In-situ imaging of Langmuir monolayers by second-harmonic microscopy," Thin Solid Films 284, 659-662 (1996).

[3] I. Freund, M. Deutsch, and A. Sprecher, "Connective tissue polarity. Optical second-harmonic microscopy, crossed-beam summation, and small-angle scattering in rat-tail tendon," Biophys. J. 50, 693-712 (1986). 
[4] P. J. Campagnola, A.C. Millard, M. Terasaki, P.E. Hoppe, C. J. Malone, and W.A. Mohler, "3Dimesional High-Resolution Second Harmonic Generation Imaging of Endogenous Structural Proteins in Biological Tissues, " Biophys J. 81, 493-508 (2002).

[5] L. Moreaux, O. Sandrea, and J. Mertz, "Membrane Imaging by Second Harmonic Generation Microscopy," J. Opt. Soc. Am. B 17, 1685-1689 (2000).

[6] D.A. Dombeck, K.A. Kasischke, H.D. Vishwasrao, M. Ingelsson, B.T. Hyman, and W.W. Webb, " Uniform polarity microtubule assemblies imaged in native brain tissue by secondharmonic generation microscopy," Proc. Natl. Acad. Sci. U.S.A. 100, 7081 (2003).

[7] M. Strupler, M. Hernest, C. Fligny, J. L. Martin, P.-L. Tharaux, and M. C. SchanneKlein, "Second Harmonic Microscopy to Quantify Renal Interstitial Fibrosis and Arterial Remodeling," J. Biomed. Optics 13, 054041 (2008).

[8] O. Nadiarnykh, R. LaComb, M. Brewer, and P.J. Campagnola, "Second Harmonic Generation Imaging Microscopy of Ovarian Cancer,” Biophys. J. 94, 4504 - 4514 (2008).

[9] E. Ralston, B. Swaim, M. Czapiga, W.L. Hwu, Y.H. Chien, M.G. Pittis, B. Bembi, O. Schwartz, P. Plotz, and N. Raben, "Detection and imaging of non-contractile inclusions and sarcomeric anomalies in skeletal muscle by second harmonic generation combined with twophoton excited fluorescence," J. Struct. Biol. 162, 500-508 (2008).

[10] D. Axelrod, "Carbocyanine dye orientation in red cell membrane studied by mi-croscopic fluorescence polarization," Biophys. J. 26, 557-574 (1979).

[11] J. Borejdo and S. Burlacu, "Measuring Orientation of Actin Filaments within a Cell: orientation of Actin in Intestinal Microvilli," Biophys. J. 65, 300-309 (1993).

[12] M. Flörsheimer, M. Bösch, C. Brillert, M. Wierschem, and H. Fuchs, "Second-harmonic microscopy - a quantitative probe for molecular surface order," Adv. Mater. 9, 10611065 (1997).

[13] V. Le Floc'h, S. Brasselet, J.-F. Roch, and J. Zyss, "Monitoring of orientation in molecular ensembles by polarization sensitive nonlinear microscopy," J. Phys.Chem. B 107, 12403-12410 (2003).

[14] C. Anceau, S. Brasselet, and J. Zyss, "Local orientational distribution of molecular monolayers probed by nonlinear microscopy," Chem. Phys. Lett. 411, 98-102 (2005).

[15] S. Brasselet, V. Le Floc'h, F. Treussart, J.-F. Roch, J. Zyss, E. Botzung-Appert, and A. Ibanez, "In situ diagnostics of the crystalline nature of single organic nanocrystals by nonlinear microscopy," Phys. Rev. Lett. 92, 207401-204405 (2004). 
[16] P. Stoller, B.M. Kim, A.M. Rubenchik, K.M. Reiser, and L.B. Da Silva, "Polarizationdependent optical second harmonic imaging of a rat-tail tendon," J. Biomed. Opt. 7, 205-214 (2000).

[17] R. M. Williams, W. R. Zipfel, and W. W. Webb, "Interpreting Second-Harmonic Generation Images of Collagen I Fibrils," Biophys. J. 88, 13771386 (2005).

[18] Yen, S., Chen, W. L., Wu, H. Y., Li, F. C., Lo, W., Lin, S. J., Jee, S. H., Chen, Y. F., So, P. T. C., and Dong, C. Y., "Collagen Thermal denaturation study with high resolution second harmonic generation imaging and polarization optical microscopy," Biophys. J. 91, 2620 2625 (2006).

[19] F.Tiaho, G. Recher, and D. Rouède, "Estimation of helical angles of myosin and collagen by second harmonic generation imaging microscopy," Opt. Expr. 15, 4054-4065 (2007).

[20] C. Odin, Y. Le Grand, A. Renault, L. Gailhouste, and G. Baffet, "Orientation fields of nonlinear biological fibrils by second harmonic generation microscopy," J. Microsc. 229, 32-38 (2008).

[21] S. Psilodimitrakopoulos, S. I. C. O. Santos, I. Amat-Roldan, A. K. N. Thayil, D. Artigas, and P. Loza-Alvarez, "In vivo, pixel-resolution mapping of thick filaments' orientation in nonfibrilar muscle using polarization-sensitive second harmonic generation microscopy," J. Biomed. Opt. 14, 014001 (2009).

[22] P. Refregier, M. Roche, and S. Brasselet, "Precision analysis in polarization-resolved second harmonic generation microscopy," Opt. Lett. 36, 2149-2151 (2011).

[23] M. G. Kuzyk, K. D. Singer, H. E. Zahn, and L. A. King, "Second-order nonlinear-optical tensor properties of poled films under stress," J. Opt. Soc. Am. B 6, 742-752 (1989).

[24] R. Bersohn, Y.-H. Pao, and H. L. Frisch, "Double-Quantum Light Scattering by Molecules," J. Chem. Phys. 45, 3184-3198 (1966).

[25] S. Kielich, "Molecular interactions in optically induced nonlinearities," IEEE J. Quantum Electron., QE-4, 744-752 (1968).

[26] J. Zyss and J. L. Oudar, "Relations between microscopic and macroscopic lowest-order optical nonlinearities of molecular crystals with one- or two-dimensional units," Phys. Rev. A 26, 2028-2048 (1982).

[27] A. Deniset-Besseau, J. Duboisset, E. Benichou, F. Hache, P.-F. Brevet, and M. C. SchanneKlein, "Measurement of the second order hyperpolarizability of the collagen triple helix and 
determination of its physical origin," J. Phys. Chem. B 113, 13437 (2009).

[28] P. Schön, F. Munhoz, A. Gasecka, S. Brustlein, S. Brasselet, "Polarization distortion effects in polarimetric two-photon microscopy," Opt. Expr. 16, 20891-20901 (2008).

[29] P. Schön, M. Behrndt, D. Ait-Belkacem, H. Rigneault, S. Brasselet, "Polarization and Phase Pulse Shaping applied to Structural Contrast in Nonlinear Microscopy Imaging," Phys. Rev. A 81, 013809 (2010).

[30] J. Zyss, "Molecular engineering implications of rotational invariance in quadratic nonlinear optics: From dipolar to octupolar molecules and materials," J. Chem. Phys. 98, 6583-6600 (1993).

[31] S. Brasselet, "Polarization resolved nonlinear microscopy: application to structural molecular and biological imaging," Advances in Optics and Photonics 3, 205-271 (2011).

[32] I. Amat-Roldan, S. Psilodimitrakopoulos, P. Loza-Alvarez, and D. Artigas, "Fast image analysis in polarization SHG microscopy," Opt. Express 18, 17209-17219 (2010).

[33] S. Brasselet and J. Zyss, "Control of the polarization dependence of optically poled nonlinear polymer films," Opt. Lett. 22, 1464 (1997).

[34] D. Aït-Belkacem, A. Gasecka, F. Munhoz, S. Brustlein, and S. Brasselet, "Influence of birefringence on polarization resolved nonlinear microscopy and collagen SHG structural imaging," Opt. Express 18, 14859-14870 (2010).

[35] N. Fourre, E. Millerot-Serrurot, R. Garnote, J.-M. Zahm, N. Bonnet, J.-M. Millot, P. Jeannesson, "Extracellular matrix proteins protect human HT1080 cells against the antimigratory effect of doxorubicin," Cancer Science 99, 1699-1705 (2008)

[36] I. Gusachenko, G. Latour, and M.-C. Schanne-Klein, "Polarization-resolved Second Harmonic microscopy in anisotropic thick tissues," Opt. Express 18, 19339-19352 (2010) 